\title{
An epidemiological investigation of Campylobacter in pig and poultry farms in the Mekong delta of Vietnam
}

\author{
J. J. CARRIQUE-MAS ${ }^{1,2} *$ J. E. BRYANT ${ }^{1,2}$, N. V. CUONG ${ }^{1}$, N. V. M. HOANG ${ }^{1}$, \\ J. CAMPBELL ${ }^{1}$, N. V. HOANG ${ }^{3}$, T. T. N. DUNG ${ }^{1}$, D.T. DUY ${ }^{1}$, N. T. HOA ${ }^{1,2}$, \\ C. THOMPSON ${ }^{1,2}$, V. V. HIEN ${ }^{3}$, V. V. PHAT ${ }^{1}$, J. FARRAR ${ }^{1,2}$ AND S. BAKER $^{1,2,4}$ \\ ${ }^{1}$ The Hospital for Tropical Diseases, Wellcome Trust Major Overseas Programme, Oxford University Clinical \\ Research Unit, Ho Chi Minh City, Vietnam \\ ${ }^{2}$ Centre for Tropical Medicine, Nuffield Department of Clinical Medicine, Oxford University, UK \\ ${ }^{3}$ Sub-Department of Animal Health, Dong Thap Province, Vietnam \\ ${ }^{4}$ The London School of Hygiene and Tropical Medicine, London, UK
}

Received 21 March 2013; Final revision 25 June 2013; Accepted 1 September 2013; first published online 25 September 2013

\section{SUMMARY}

Campylobacter are zoonotic pathogens commonly associated with gastroenteritis. To assess the relevance of Campylobacter in Vietnam, an economically transitioning country in SE Asia, we conducted a survey of 343 pig and poultry farms in the Mekong delta, a region characterized by mixed species farming with limited biosecurity. The animal-level prevalence of Campylobacter was $31.9 \%, 23.9 \%$ and $53.7 \%$ for chickens, ducks and pigs, respectively. C. jejuni was predominant in all three host species, with the highest prevalence in pigs in high-density production areas. Campylobacter isolates demonstrated high levels of antimicrobial resistance ( $21 \%$ and $100 \%$ resistance against ciprofloxacin and erythromycin, respectively). Multilocus sequence type genotyping showed a high level of genetic diversity within $C$. jejuni, and predicted C. coli inter-species transmission. We suggest that on-going intensification of animal production systems, limited biosecurity, and increased urbanization in Vietnam is likely to result in Campylobacter becoming an increasingly significant cause of human diarrhoeal infections in coming years.

Key words: Campylobacter infections, epidemiology, Vietnam, zoonoses.

\section{INTRODUCTION}

Campylobacter spp. are ubiquitous foodborne pathogens [1], and among the most common cause of bacterial gastroenteritis in low-, middle- and highincome countries [2,3]. The global distribution of Campylobacter is attributed to its universal ability to colonize and form part of the commensal flora of

\footnotetext{
* Author for correspondence: Dr J. J. Carrique-Mas, Oxford University Clinical Research Unit, 764 Vo Van Kiet, District 5, Ho Chi Minh City, Vietnam.

(Email: jcarrique-mas@oucru.org)
}

a wide range of species including poultry [4], pigs [5] and cattle [6]. The epidemiology of symptomatic Campylobacter infections in humans reflects the complexity and variability of sources of Campylobacter. Risk factors for infection include the consumption of undercooked meat, unpasteurized dairy products or contaminated drinking water, and direct contact with farm animals [7-9]. Of all Campylobacter spp., Campylobacter jejuni and Campylobacter coli are the two most common causes of human infection [10]. Globally, C. jejuni is more prevalent in poultry, whereas $C$. coli is more common in pigs $[5,11,12]$. Poultry meat is considered to be the primary source 
of human Campylobacter infection; this is particularly well established for $C$. jejuni [7, 12-14].

Diarrhoeal disease is common in Vietnam; community-based studies indicate a moderately high burden in children aged $<5$ years (about $1-3$ cases/ child per year), which is higher than typical levels from developed countries $[15,16]$. Campylobacter is likely to play a significant role, with some aetiological investigations indicating that Campylobacter may be responsible for $2-7 \%$ of cases of gastroenteritis in children aged $<5$ years $[15,17]$. Notably, and in common with other developing countries, unpublished data suggests that Campylobacter-induced diarrhoea is relatively rare in adult populations [3]. Limited data on species identification on 88 isolates from cases of clinical diarrhoea from 1996 to 1999 indicate that $85 \%$ were $C$. jejuni, and the remainder $C$. coli [15]. Previous surveys of fresh chicken samples collected from retail markets and canteens in Vietnam have indicated a Campylobacter prevalence of $28-31 \%$ $[18,19]$. However, none of 75 pork and beef samples collected from the same sites tested positive for Campylobacter [18].

To date there are no published studies regarding the prevalence or distribution of Campylobacter spp. in animal reservoirs in Vietnam, where mixed species farming occurs with low levels of biosecurity. We aimed to determine the prevalence of Campylobacter in the main species farmed in the Mekong delta of Vietnam by surveying 343 pigs and poultry farms in Dong Thap province. Risk factors that may explain the presence of Campylobacter at the individualanimal level were investigated, and the isolated organisms were genotyped and evaluated for antimicrobial susceptibility to determine their circulation and potential threat to human health.

\section{MATERIALS AND METHODS}

\section{Study location}

The survey was conducted between February and May 2012 in Dong Thap province, Vietnam, a rural province located in the Mekong delta between latitude $10^{\circ} 58^{\prime} 01 \cdot 70^{\prime \prime}$ and $10^{\circ} 07^{\prime} 36.56^{\prime \prime} \mathrm{N}$ and longitude $105^{\circ} 56^{\prime} 47 \cdot 22^{\prime \prime}$ and $105^{\circ} 11^{\prime} 16 \cdot 30^{\prime \prime}$. Covering an area of $3238 \mathrm{~km}^{2}$, the province is home to about 1.6 million people, 3.1 million ducks, 1.3 million chickens, 274000pigs, and 22000 cattle and buffalo. The study included 4/12 districts (Cao Lanh, Chau Thanh, Hong Ngu, Thanh Binh) (Fig. 1).

\section{Survey design}

We aimed to collect samples from 612 animals from 306 clusters (farms) (i.e. two animals per farm). Such sample size would theoretically allow the detection of an overall $35 \%$ prevalence with an estimated relative error of $10 \%$ and $80 \%$ confidence level, assuming a design effect of 2 (i.e. low-level clustering). Based on a farm census provided by the Dong Thap Sub-Department of Animal Health (sDAH), farms were stratified by district $(X=4)$, animal species ( $Y=3$; chickens, ducks, pigs) and size $(Z=3$; small, medium, large). The cut-offs for animal numbers that determined farm size were as follows: for chickens 20-50 (small), 51-100 (medium) and >100 (large); for ducks 50-200 (small); 201-1000 (medium) and >1000 (large); and for pigs 5-20 (small), 21-50 (medium) and $>50$ (large). Each farm was defined by the species of animal that represented the highest source of income to the farmer, and was included in only one species category. The total number of strata was, therefore, $X^{*} Y^{*} Z=36$. We aimed to sample ten randomly selected farms from each stratum and each farm was sampled on a single occasion. From each farm faecal samples from 1-3 individual animals were collected, except from a subset of 20 farms in Cao Lanh district, where more intensive sampling was performed (10-14 animals per farm). About $5 \mathrm{~g}$ freshly voided faeces were aseptically collected from each animal and placed immediately into sterile $25 \mathrm{ml}$ universal tubes. Samples were kept at $4{ }^{\circ} \mathrm{C}$ and were transported to the laboratory within $24 \mathrm{~h}$, where culture was immediately initiated.

\section{Laboratory methods}

Fresh faecal samples were incubated on Campylobacter selective agar (Blood Free with CCDA supplement) (Oxoid Ltd, UK) for $48 \mathrm{~h}$ at $42{ }^{\circ} \mathrm{C}$ within a gas jar with microaerophilic conditions using CampyGen gas packs (Oxoid Ltd, UK). Colonies from these plates were screened by Gram stain for Gram-negative Vibrio-like bacteria. Differentiation between $C$. jejuni and $C$. colilC. lari was done by hypurate test [20] and by PCR (see below). All isolates were antimicrobial susceptibility tested against ampicillin, chloramphenicol, gentamycin, sulfamethoxazole-trimethoprim, nalidixic acid, ofloxacin, ciprofloxacin, clindamycin and erythromycin using the Kirby-Bauer (disk diffusion) method on Muller-Hinton Agar (Oxoid, UK) and interpreted using CLSI guidelines for Enterobacteriaceae (www.clsi.org). 
(a)

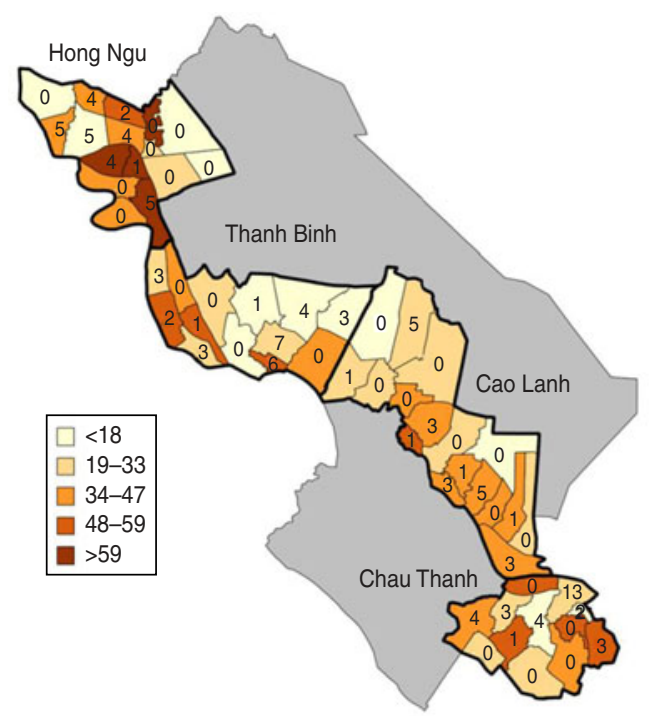

$(c)$

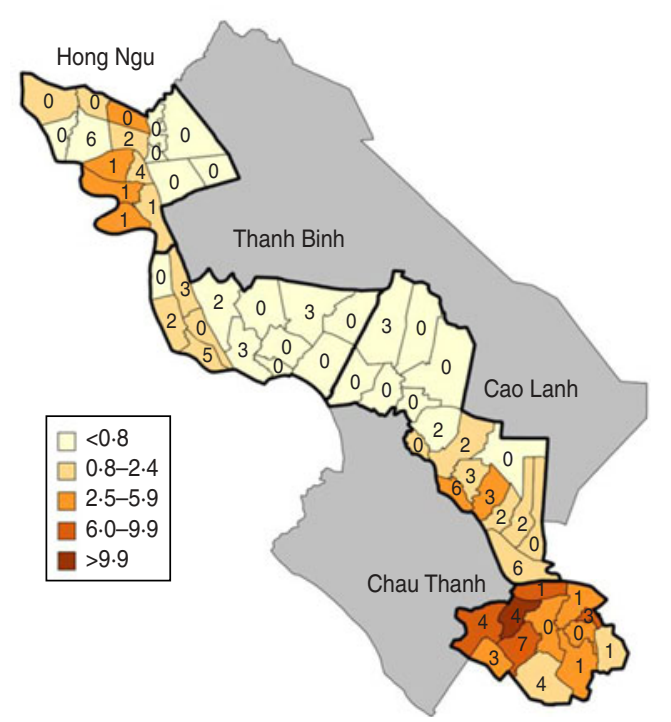

(b)

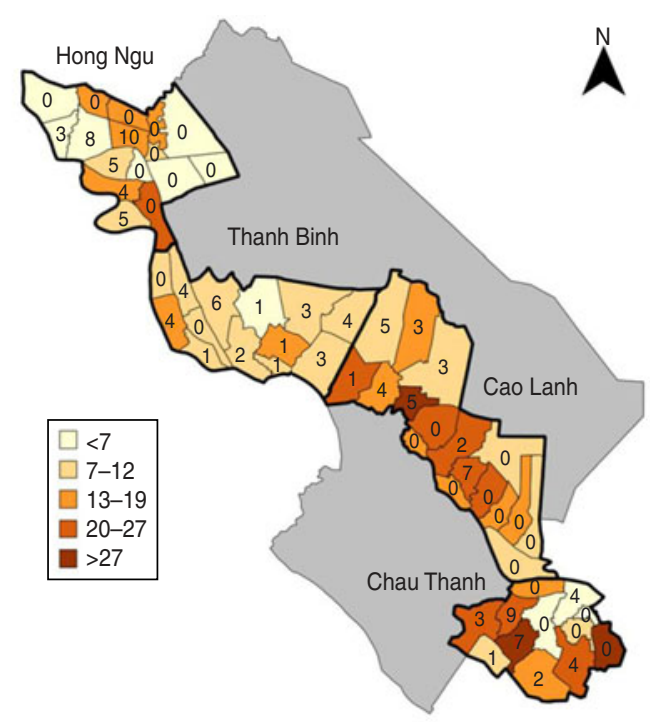

(d)

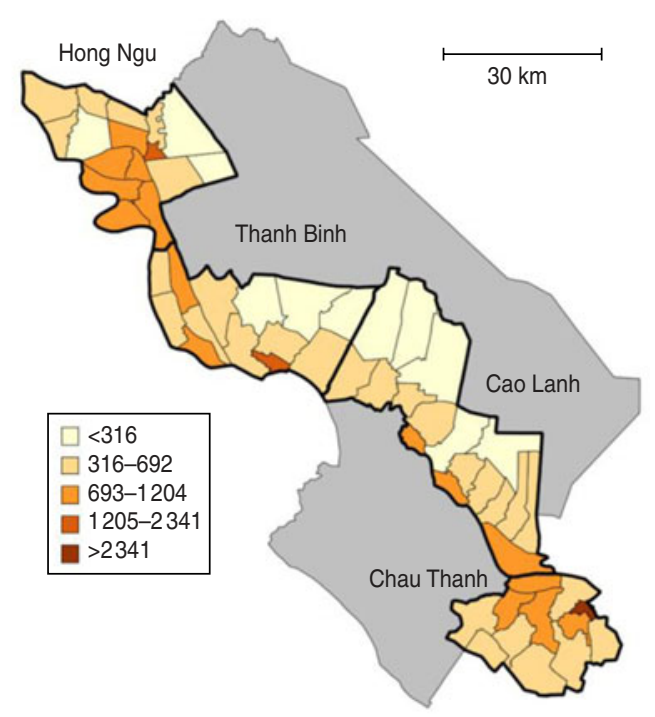

Fig. 1. The geographical distribution of chicken, duck and pig farms and human population density in Dong Thap province, Mekong delta, Vietnam. Maps showing Dong Thap province (total area), highlighting the four study districts (Hong Ngu, Thanh Binh, Cao Lanh, Chau Thanh) and communes (smallest shaded areas). (a) The density of chicken farms (per $\mathrm{km}^{2}$, as shown by shading in key) and the number of farms sampled per commune (numeral within shaded area). (b) The density of duck farms (per $\mathrm{km}^{2}$, as shown by shading in key) and the number of farms sampled per commune (numeral within shaded area). (c) The density of pig farms (per $\mathrm{km}^{2}$, as shown by shading in key) and the number of farms sampled per commune (numeral within shaded area). (d) Human population density per km ${ }^{2}$, as shown by shading in key).

\section{Molecular speciation and multi locus sequence typing (MLST)}

C. coli and $C$. jejuni were distinguished using PCR primers targeting the hipuricase gene (hipO) for $C$. jejeni and the aspartokinase gene (asp) for $C$. coli as described previously [21, 22]. MLST was performed on DNA extracted using the wizard genomic DNA extraction kit (Promega, USA) from one full loop of pure Campylobacter colonies of overnight culture in blood agar media. PCR amplification was performed on the seven MLST loci [aspA (aspartase), $g \ln A$ (glutamine synthetase), glt $A$ (citrate synthase), gly $A$ (serine hydroxy methyl transferase), tkt (transketolase), 
pgm (phospho glucomutase) and unc A (ATP synthase alpha subunit)], as described previously [23]. PCR products were purified using QIAquick column-based PCR purification system (Qiagen) and sequenced in forward and reverse directions using the Big Dye cycle sequencing kit (Applied Biosystems, USA) on an ABI 3770 automatic sequencer according to the manufacturer's instructions. Minimum spanning trees were created from the resulting MLST data using bionumerics (Applied Mathematics, Belgium).

\section{Statistical analyses}

Separate analyses were performed for chickens, ducks and pigs. District- and species-specific estimates of prevalence were calculated before and after adjusting for the sampling frame that implied a known (but non-random) probability of an animal being selected. This was performed by assigning a sample weight $(1 / W)$ to each observation [24], where $W=P(\mathrm{~S}) \times P$ $(\mathrm{F}) \times P(\mathrm{~A})$, and $P(\mathrm{~S})=$ probability of a stratum being selected (since the size strata were determined arbitrarily); $P(\mathrm{~F})=$ probability of a farm being selected from each stratum (since strata contained different numbers of farms); $P(\mathrm{~A})=$ probability of an animal being selected from a selected farm (since a variable number of animals were sampled from each farm).

Clustering of infection within farms was investigated using the subset of samples $(N=244)$ from intensively sampled farms $(N=20)$ in Cao Lanh district. The intra-class correlation coefficient (ICC) was calculated separately for each host species (chickens, ducks, pigs) for Campylobacter spp., C. jejuni and C. coli using a one-way random-effects model [25].

Survey data on animal and farm characteristics were collected using validated questionnaires and entered into an Access database (Microsoft, USA). To explore potential risk factors for Campylobacter infection, two-level random-effects logistic regression models (animals nested within farms) were built to account for the variable numbers of animals sampled from each farm [24]. The outcomes modelled were: all Campylobacter spp., C. jejuni, and C. coli. Models were built separately for each animal species and outcome (total nine models). Variables investigated as potential risk factors included farm-level descriptive management variables (number of flocks, number of animals, type of water, use of antibiotics), density of farms and humans at the commune level, district and other individual animal-level variables (age, presence of diarrhoea). Variables were considered as candidates for multivariable analysis based on their biological plausibility and a $P$ value $<0 \cdot 15$ in the univariable analysis. Candidate variables were ranked by their degree of significance and were included in the model starting with the most significant ones using a step-wise forward approach [26]. In the final multivariable model for each species, variables were retained if their $P$ value was $<0.05$ for any of the three outcome variables. The suitability of each new variable included in the model was assessed using Akaike's Information Criterion [27]. All statistical analyses were performed using $\mathrm{R}$ (packages Epicalc and EpiR; http://www.r-project.org). Random-effects logistic regression modelling was performed using the lme4 package. Maps were produced using Quantum GIS v. 1·7·4 (http://qgis.osgeo.org).

\section{RESULTS}

\section{Baseline farm data}

A total of 343 farms distributed across Dong Thap province were sampled (Fig. 1), and 634 faecal specimens were collected and tested for the presence of Campylobacter. The number of samples collected and analysed from each host species (pig, chicken, duck) and the number of farms sampled are summarized in Supplementary Table S1 (available online). The distribution of the farms sampled by district within Dong Thap province, and the underlying farm density and human population density, are shown in Figure 1. The district of Chau Thanh contained over $70 \%$ of the pig production in the study area. In two of the four surveyed districts, there were few pig farms in the large size stratum, and all farms were sampled. Notably, the sampling fraction was considerably higher in the larger farms. Across the study area chicken farms had the greatest density $\left(37.9 \mathrm{farms} / \mathrm{km}^{2}\right)$ followed by duck farms $\left(12.5 \mathrm{farms} / \mathrm{km}^{2}\right)$ and pig farms $\left(1.3 \mathrm{farms} / \mathrm{km}^{2}\right)$. The density of pig farms was greatest in Chau Thanh district $\left(3.6 \mathrm{farms} / \mathrm{km}^{2}\right)$ and lowest in Thanh Binh district $\left(0 \cdot 5 \mathrm{farms} / \mathrm{km}^{2}\right)$ (Table 1).

\section{Adjusted estimates of Campylobacter prevalence}

A total of 207 isolates were recovered from 202 Campylobacter-positive animals (five pigs tested positive for both $C$. jejuni and $C$. coli). Two isolates (1\%) could not be identified as either $C$. jejuni or $C$. coli. The overall unadjusted prevalence of Campylobacter from faecal specimens of chickens, ducks and pigs 
Table 1. Human population and farm statistics (per $\mathrm{km}^{2}$ ) for four districts of Dong Thap province, Mekong delta, Vietnam

\begin{tabular}{|c|c|c|c|c|c|c|c|c|}
\hline \multirow[b]{2}{*}{ District } & \multicolumn{2}{|l|}{ Human } & \multicolumn{2}{|c|}{ Chicken farms } & \multicolumn{2}{|c|}{ Duck farms } & \multicolumn{2}{|c|}{ Pig farms } \\
\hline & Median & IQR & Median & IQR & Median & IQR & Median & IQR \\
\hline Cao Lanh & 515 & $341-647$ & $36 \cdot 1$ & $29 \cdot 5-39 \cdot 8$ & $16 \cdot 5$ & $12 \cdot 4-21 \cdot 5$ & $1 \cdot 1$ & $0 \cdot 4-1 \cdot 6$ \\
\hline Chau Thanh & 623 & $522-835$ & $34 \cdot 4$ & $21 \cdot 9-51 \cdot 1$ & $16 \cdot 1$ & $18 \cdot 0-25 \cdot 7$ & $3 \cdot 6$ & $2 \cdot 2-8 \cdot 0$ \\
\hline Hong Ngu & 725 & $545-906$ & $43 \cdot 8$ & $40 \cdot 1-66 \cdot 0$ & $11 \cdot 9$ & $5 \cdot 3-13 \cdot 9$ & $1 \cdot 4$ & $1 \cdot 0-2 \cdot 8$ \\
\hline Thanh Binh & 594 & $389-668$ & $31 \cdot 6$ & $17 \cdot 6-58 \cdot 3$ & $9 \cdot 7$ & $7 \cdot 9-11 \cdot 3$ & $0 \cdot 5$ & $0 \cdot 2-0 \cdot 8$ \\
\hline All & 616 & $428-778$ & $37 \cdot 9$ & $23 \cdot 0-45 \cdot 1$ & $12 \cdot 5$ & $8 \cdot 4-17.9$ & $1 \cdot 3$ & $0 \cdot 5-2 \cdot 4$ \\
\hline
\end{tabular}

IQR, Interquartile range.

(a)
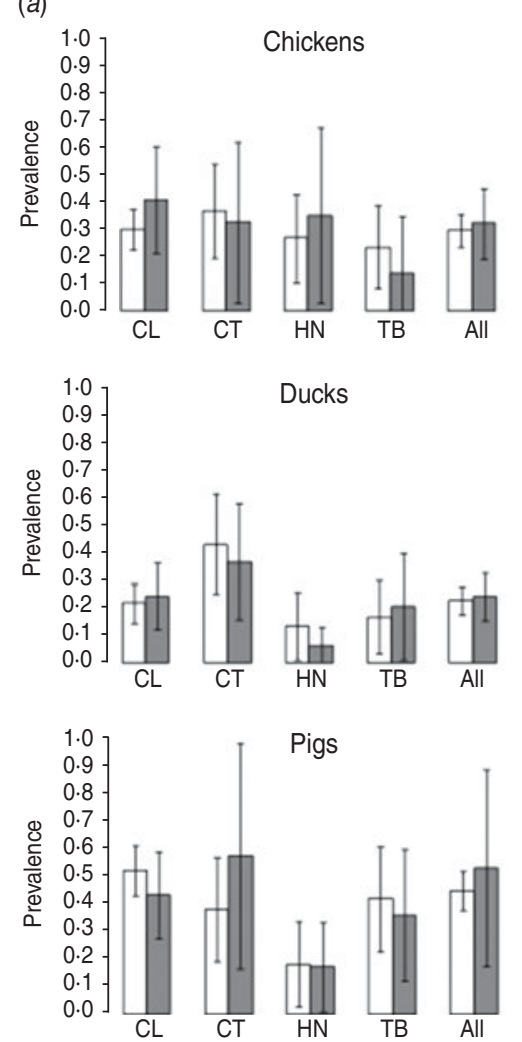

(b)
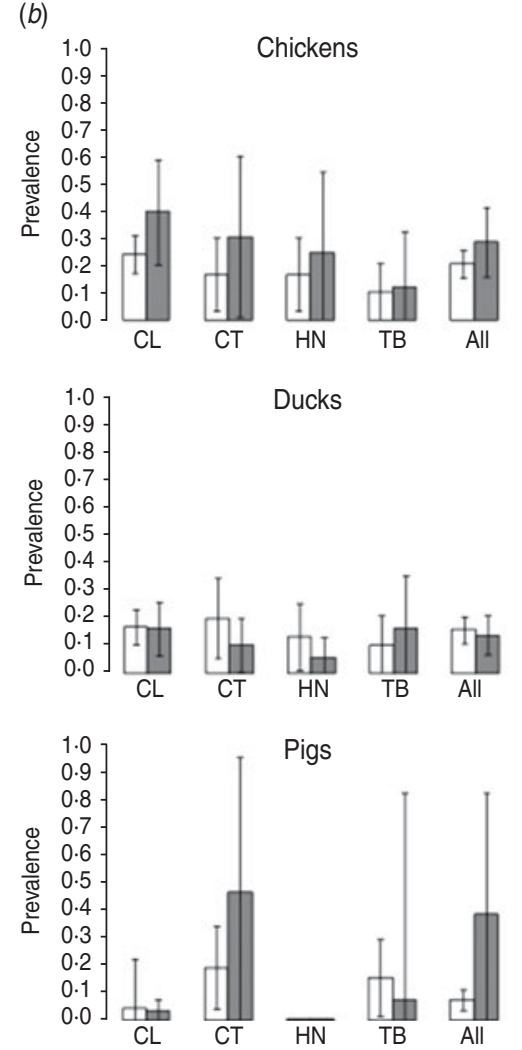

(c)
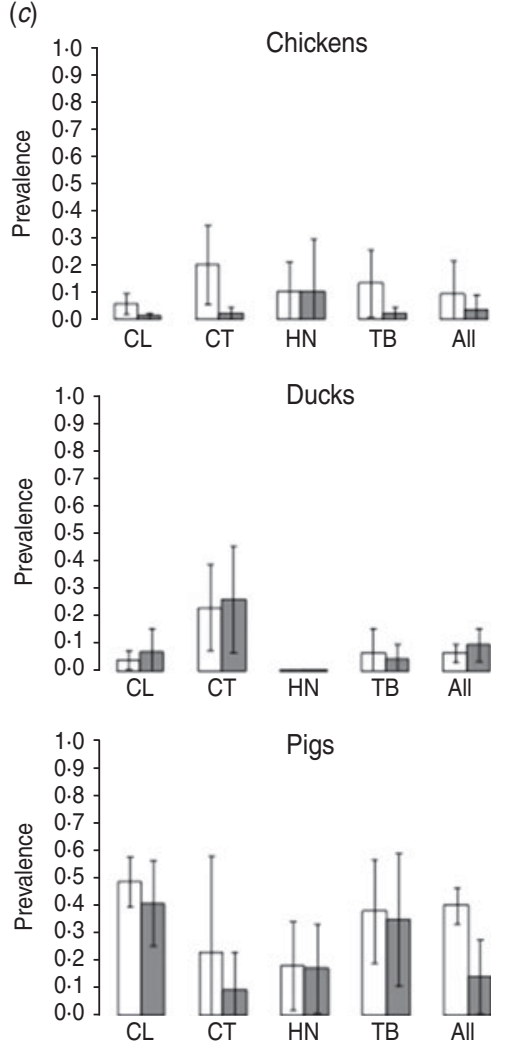

Fig. 2. The prevalence of Campylobacter by district in Dong Thap province, Mekong delta, Vietnam. Bar charts showing the unadjusted (white bars) and adjusted (grey bars) prevalence of (a) Campylobacter spp., (b) C. jejuni and (c) C. coli in chickens, ducks and pigs in Cao Lanh (CL), Chau Thanh (CT), Hong Nhu (HN) and Thanh Binh (TB) districts in Dong Thap province.

was $29 \cdot 4 \%, 22 \cdot 5 \%$ and $44 \cdot 9 \%$, respectively. The adjusted prevalence was $31.9 \%$ [95\% confidence interval (CI) 19.0-44.8] in chickens, $23.9 \%$ (95\% CI $15 \cdot 1-32 \cdot 7)$ in ducks, and $53 \cdot 7 \%(95 \%$ CI $17 \cdot 6-89 \cdot 7)$ in pigs. The adjusted prevalence of $C$. jejuni in chickens, ducks and pigs was $28 \cdot 4 \%, 13 \cdot 8 \%$ and $38 \cdot 6 \%$, respectively, whereas the adjusted prevalence of C. coli in chickens, ducks and pigs was $3 \cdot 5 \%, 9 \cdot 7 \%$ and $14 \cdot 1 \%$, respectively (Fig. 2). In chickens, the highest crude prevalence $(45 \%)$ was reported in the 10-24 weeks age group. The high adjusted prevalence of $C$. jejuni in pigs was a consequence of the large sampling weight applied to pigs from large herds in Chau Thanh district, which had a notably higher prevalence of $C$. jejuni $(47 \%)$ compared to other districts $(6 \%)$. Clustering of Campylobacter infection by farm was investigated 
Table 2. The animal- and farm-level prevalence of Campylobacter on 20 farms in Cao Lanh district, Dong Thap province, Mekong delta (Vietnam)

\begin{tabular}{|c|c|c|c|c|c|c|c|c|c|}
\hline \multirow[b]{2}{*}{ Species } & \multicolumn{3}{|c|}{ Campylobacter spp. } & \multicolumn{3}{|l|}{ C. jejuni } & \multicolumn{3}{|l|}{ C. coli } \\
\hline & Animal & Farm & ICC & Animal & Farm & ICC & Animal & Farm & ICC \\
\hline Chickens & $\begin{array}{l}24 / 100 \\
(24 \cdot 0 \%)\end{array}$ & $\begin{array}{l}7 / 8 \\
(87 \cdot 5 \%)\end{array}$ & $0 \cdot 053$ & $\begin{array}{l}20 / 100 \\
(20 \cdot 0 \%)\end{array}$ & $\begin{array}{l}6 / 8 \\
(75 \cdot 0 \%)\end{array}$ & $0 \cdot 043$ & $\begin{array}{l}4 / 100 \\
(4 \cdot 0 \%)\end{array}$ & $\begin{array}{l}3 / 8 \\
(37 \cdot 5 \%)\end{array}$ & $<0 \cdot 001$ \\
\hline Ducks & $\begin{array}{l}15 / 83 \\
(18 \cdot 1 \%)\end{array}$ & $\begin{array}{l}5 / 7 \\
(71 \cdot 4 \%)\end{array}$ & $0 \cdot 178$ & $\begin{array}{l}11 / 83 \\
(13 \cdot 3 \%)\end{array}$ & $\begin{array}{l}5 / 7 \\
(71 \cdot 4 \%)\end{array}$ & $0 \cdot 153$ & $\begin{array}{l}3 / 83 \\
(3 \cdot 6 \%)\end{array}$ & $\begin{array}{l}2 / 7 \\
(28 \cdot 5 \%)\end{array}$ & $0 \cdot 028$ \\
\hline Pigs & $\begin{array}{l}35 / 61 \\
(57 \cdot 4 \%)\end{array}$ & $\begin{array}{l}5 / 5 \\
(100 \%)\end{array}$ & $0 \cdot 196$ & $\begin{array}{l}2 / 61 \\
(3 \cdot 3 \%)\end{array}$ & $\begin{array}{l}2 / 5 \\
(40 \%)\end{array}$ & $0 \cdot 015$ & $\begin{array}{l}33 / 61 \\
(54 \cdot 1 \%)\end{array}$ & $\begin{array}{l}5 / 5 \\
(100 \%)\end{array}$ & $0 \cdot 192$ \\
\hline
\end{tabular}

ICC, Intra-class correlation coefficient.

Table 3. Variables investigated for their potential association with Campylobacter infection, and summary of their descriptive values in animals sampled in Dong Thap province, Mekong delta, Vietnam

\begin{tabular}{llll}
\hline \hline Variable & $\begin{array}{l}\text { Chickens } \\
(n=231)\end{array}$ & $\begin{array}{l}\text { Ducks } \\
(n=215)\end{array}$ & $\begin{array}{l}\text { Pigs } \\
(n=188)\end{array}$ \\
\hline Median, no. of animals target species in the farm (IQR) & $90(48-200)$ & $580(178-783)$ & $40(19-63)$ \\
Median no. of flocks per farm (poultry) (IQR) & $2(1-2)$ & $2(1-2)$ & - \\
Median of average age (weeks) (IQR) & $10(5 \cdot 5-30)$ & $20(4-52)$ & $17(10-31)$ \\
Confined (\%) & $69 \cdot 3$ & $11 \cdot 7$ & 100 \\
Presence of chickens in the farm (\%) & 100 & $27 \cdot 6$ & $60 \cdot 0$ \\
Presence of ducks in the farm (\%) & $5 \cdot 3$ & 100 & $24 \cdot 6$ \\
Presence of pigs in the farm (\%) & $21 \cdot 6$ & $14 \cdot 0$ & 100 \\
Multi-age (poultry farms) (\%) & $46 \cdot 4$ & $0 \cdot 5$ & - \\
Diarrhoea (\%) & $24 \cdot 7$ & $42 \cdot 5$ & $12 \cdot 8$ \\
Use of water well (\%) & $3 \cdot 8$ & $25 \cdot 0$ \\
Use of water from natural waterways (\%) & $80 \cdot 2$ & $90 \cdot 7$ & $58 \cdot 8$ \\
Use of municipal water (\%) & $18 \cdot 9$ & $6 \cdot 5$ & $20 \cdot 8$ \\
Use of antibiotics in last 2 months (\%) & $78 \cdot 8$ & $76 \cdot 1$ & $94 \cdot 1$ \\
Use of commercial feed (\%) & $78 \cdot 0$ & $90 \cdot 6$ & $88 \cdot 3$ \\
Cao Lanh district (\%) & $60 \cdot 8$ & $57 \cdot 5$ & $61 \cdot 0$ \\
Chau Thanh district (\%) & $13 \cdot 2$ & $14 \cdot 0$ & $13 \cdot 9$ \\
Hong Ngu district (\%) & $13 \cdot 2$ & $14 \cdot 0$ & $11 \cdot 2$ \\
Thanh Binh district (\%) & $12 \cdot 8$ & $14 \cdot 5$ & $13 \cdot 9$ \\
\hline \hline
\end{tabular}

IQR, Interquartile range.

with 244 samples from 20 farms in Cao Lanh district. Results indicated a low level of clustering of Campylobacter infection in farms, ranging from ICC $=0.196$ (the highest) in pig farms to ICC $=0.053$ (the lowest) in chicken farms (Table 2).

\section{Risk factors for Campylobacter infection in chickens, ducks and pigs}

A number of variables with plausible biological associations with Campylobacter colonization were investigated using multivariable hierarchical modelling (Table 3). A combination of animal species on farms was common; with $35 \%$ of pig farms also having chickens, and $18 \%$ of poultry farms having pigs. We found important differences in management between poultry species, with the majority of ducks $(99.5 \%)$ raised in synchronized-age flocks, compared to $53.6 \%$ of chickens, the remainder being raised in continuous mixed-age flocks. Age was investigated both as a (log-transformed) continuous variable and as a categorical variable (quintiles) in both chicken and duck models. For chickens, the age group corresponding to the third quartile (10-24 weeks) had the greatest risk and the best fit in the final models [odds ratio (OR) 3.6] (Table 4). There was an association 
Table 4. Results of models for risk factors of Campylobacter infection in chickens, ducks and pigs in Dong Thap province, Mekong delta, Vietnam

\begin{tabular}{|c|c|c|c|c|c|c|}
\hline & \multicolumn{2}{|l|}{ Campylobacter spp. } & \multicolumn{2}{|l|}{ C. jejuni } & \multicolumn{2}{|l|}{ C. coli } \\
\hline & OR $(95 \% \mathrm{CI})$ & $P$ & OR $(95 \% \mathrm{CI})$ & $P$ & OR $(95 \% \mathrm{CI})$ & $P$ \\
\hline \multicolumn{7}{|l|}{ Chickens } \\
\hline Age $10-24$ weeks & $3 \cdot 9(1 \cdot 22-10 \cdot 55)$ & $0 \cdot 020$ & $2 \cdot 46(0 \cdot 60-10 \cdot 11)$ & $0 \cdot 210$ & $3.83(0 \cdot 82-17 \cdot 95)$ & $0 \cdot 080$ \\
\hline Diarrhoea & $3 \cdot 65(1 \cdot 31-10 \cdot 21)$ & $0 \cdot 013$ & $2 \cdot 58(1 \cdot 10-6 \cdot 07)$ & $0 \cdot 984$ & $13 \cdot 56(2 \cdot 48-74 \cdot 11)$ & $0 \cdot 002$ \\
\hline \multicolumn{7}{|l|}{ Ducks } \\
\hline$>1$ flock & $2 \cdot 48(1 \cdot 05-5 \cdot 85)$ & 0.037 & $2 \cdot 40(0 \cdot 83-6 \cdot 89)$ & $0 \cdot 104$ & $2 \cdot 10(0 \cdot 51-8 \cdot 56)$ & $0 \cdot 298$ \\
\hline Log, no. of pig farms $/ \mathrm{km}^{2}$ & $1 \cdot 27(0 \cdot 96-1 \cdot 69)$ & $0 \cdot 080$ & $0 \cdot 98(0 \cdot 68-1 \cdot 41)$ & 0.923 & $1 \cdot 63(1 \cdot 07-2 \cdot 48)$ & $0 \cdot 020$ \\
\hline Municipal water & $3 \cdot 80(1 \cdot 03-14 \cdot 01)$ & $0 \cdot 041$ & $5.93(1 \cdot 36-25 \cdot 89)$ & $0 \cdot 018$ & $0 \cdot 81(0 \cdot 10-6 \cdot 06)$ & $0 \cdot 836$ \\
\hline Diarrhoea & $1 \cdot 61(0 \cdot 76-3 \cdot 40)$ & $0 \cdot 211$ & $1 \cdot 09(0 \cdot 44-2 \cdot 70)$ & $0 \cdot 853$ & $4 \cdot 39(1 \cdot 13-2 \cdot 48)$ & $0 \cdot 032$ \\
\hline \multicolumn{7}{|l|}{ Pigs } \\
\hline Log age (months) & $0.59(0.38-0.94)$ & 0.026 & $0.83(0.43-1.64)$ & 0.590 & $0.57(0.35-0.94)$ & $0 \cdot 027$ \\
\hline Chau Thanh & $1 \cdot 32(0 \cdot 33-5 \cdot 22)$ & $0 \cdot 690$ & $6 \cdot 48(1 \cdot 59-26 \cdot 40)$ & 0.009 & $0 \cdot 42(0 \cdot 08-2 \cdot 29)$ & $0 \cdot 322$ \\
\hline
\end{tabular}

OR, Odds ratio; CI, confidence interval.

between $C$. coli and the occurrence of diarrhoea in both chickens and ducks (OR $13 \cdot 6$ and $4 \cdot 4$, respectively), but not in pigs. Risk factors for Campylobacter in ducks included the presence of more than one flock per farm and the use of municipal water. The use of municipal water for ducks was specifically associated with increased risk of $C$. jejuni, but not $C$. coli. The model also showed that older pigs had reduced risk of $C$. coli, with estimates indicating that a pig aged 5.5 months (third quartile) has an OR of 0.19 compared to a pig aged 2.5 months (first quartile) for Campylobacter infection. Notably, pigs in Chau Thanh had increased risk of $C$. jejuni (OR 6.5) compared to other districts.

\section{Antimicrobial susceptibility testing}

A summary of the antimicrobial susceptibility patterns and resistance prevalence for all 202 Campylobacter isolates is shown in Figure 3. Of the eight antimicrobials in the panel to which Campylobacter normally exhibits susceptibility, strains demonstrated resistance against a median of four antimicrobials [interquartile range (IQR) 4-5]. Notably, the proportion of strains demonstrating resistance against erythromycin was $100 \%$, while $99 \%$ of isolates exhibited resistance against sulfamethoxazole-trimethoprim, $92 \%$ against nalidixic acid and ofloxacin, and 20.8\% against ciprofloxacin. The susceptibility patterns were almost identical for $C$. jejuni and C. coli and there was no statistically significant difference in prevalence of resistance between host species. While antimicrobial resistance was common, there was no significant difference in the number of antimicrobials to which isolates were resistant based on whether farms had $(n=185)$ or had not $(n=17)$ used antimicrobials within the 2 months prior to sampling (4.59 vs. 4.41, respectively) (Kruskal-Wallis $P=0 \cdot 450$ )

\section{MLST}

One hundred and forty-three available Campylobacter isolates $(73 \mathrm{C}$. jejuni, 70 C. coli) were subjected to seven-loci MLST (Fig. 4). The Campylobacter strains (76 from chickens, 43 from pigs, and 24 from ducks) could be divided into 112 individual sequence types (53 C. jejuni, 59 C. coli), including 75 novel sequence types (Supplementary Table S2), and one major (ST828) ( $>3$ isolates), and 10 minor ( $\leqslant 3$ isolates) single allele variants (clonal) complexes. Overall, C. jejuni (Fig. 4a) demonstrated a higher level of genetic diversity than $C$. coli (Fig. 4b), with all but 16 C. coli isolates lying outside the major ST828 clonal complex. There was a strong association between the animal species of isolation and the clonal complex/Campylobacter spp., as the majority of strains isolated from pigs formed a single $C$. coli complex. However, this association was not absolute, as a number of $C$. coli isolated from ducks and chickens lay within the same clonal complex as isolates from pigs. Furthermore, four $C$. coli strains isolated from chickens belonging to sequence types 828, 966, 4939 
(a)

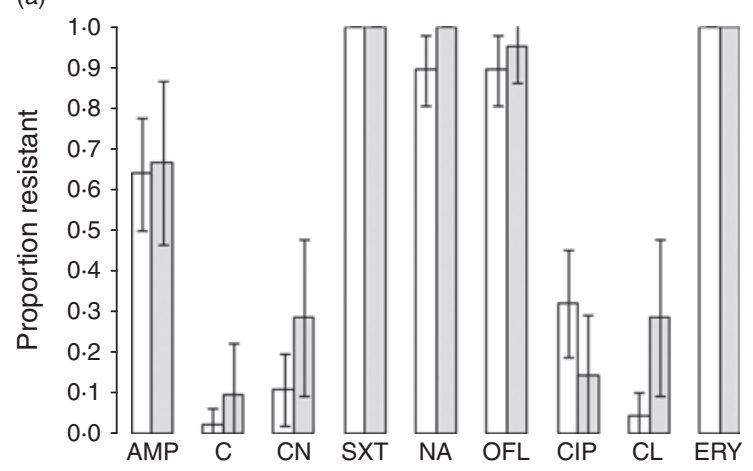

(c)

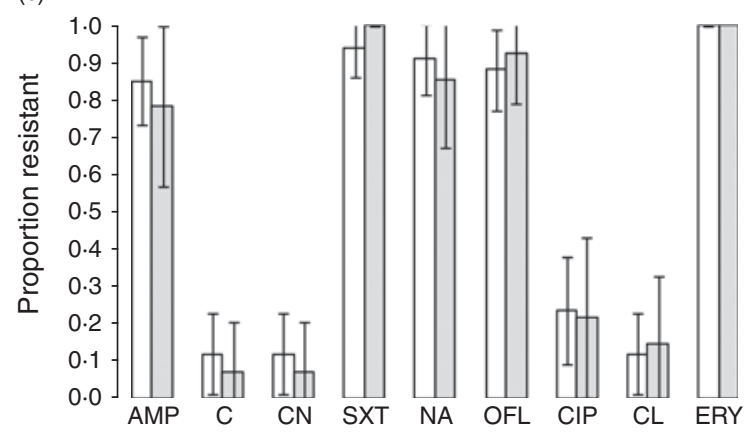

(b)

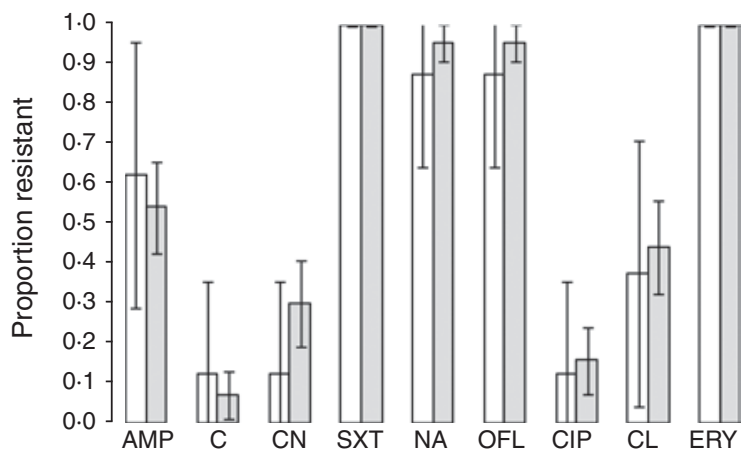

(b)

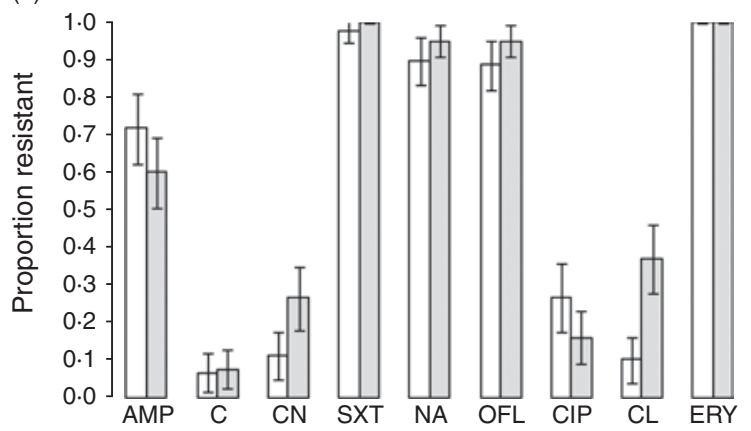

Fig. 3. The antimicrobial susceptibility profiles of Campylobacter isolated from animals in Dong Thap province, Mekong delta, Vietnam. Bar charts showing the antimicrobial susceptibility patterns of $C$. jejuni (white bars) and $C$. coli (grey bars) isolated from $(a)$ chickens (68 organisms), $(b)$ pigs ( 85 organisms), $(c)$ ducks (49 organisms) and $(d)$ all species combined (202 organisms) against 12 antimicrobials: AMP, ampicillin; C, chloramphenicol; CN, gentamycin; SXT, sulfamethoxazole-trimethoprim; NA, nalidixic acid; OFL, oxfloxacin; CIP, ciprofloxacin; CL, clindamycin; and ERY, erythromycin.

and 5935 were identical to strains identified from pigs (Fig. 4b).

\section{DISCUSSION}

This is first animal survey of its type investigating the prevalence of Campylobacter in farms in Vietnam. The key findings from this study are: (1) the predominance of $C$. jejuni as the main Campylobacter species colonizing pigs and poultry; (2) an age-related difference in Campylobacter colonization in pigs and chickens; (3) a high prevalence of resistance (>90\%) against erythromycin, nalidixic acid and ofloxacin, and moderate levels of resistance $(21 \%)$ against ciprofloxacin; (4) evidence for cross-species recombination and the transmission of $C$. coli between pigs and poultry; and (5) an association between $C$. coli infection and diarrhoea in poultry.

In spite of a low-level of biosecurity and mixing of age groups, the calculated prevalence of Campylobacter in chickens $(31.9 \%)$ was not higher than that observed in other studies in Southeast Asia (Malaysia and Thailand), where bird-level prevalence in broilers sampled in farms and wet markets has been reported to range between $64 \%$ and $76 \%$ [28-30]. However, since our study is cross-sectional, it is difficult to draw direct comparisons. With the exception of the study by Padungtod et al. [30], the other investigations were performed on chickens at slaughter age, the age at which chickens are predicted to have their highest prevalence of Campylobacter. Campylobacter infections in chickens are highly age-dependent, with a prevalence approaching $100 \%$ in broilers slaughtered after 35-42 days [31, 32]. Studies on older chickens kept for laying production have shown a decreasing prevalence after 10-20 weeks, potentially reflecting acquired immunity [33,34]. The observed highest prevalence in the 10-24 weeks age group $(45 \%)$ in our investigation is consistent with these findings, and suggests substantial differences between production systems. In Vietnam most consumed chickens are produced in backyard and semi-intensive systems, 
(a)

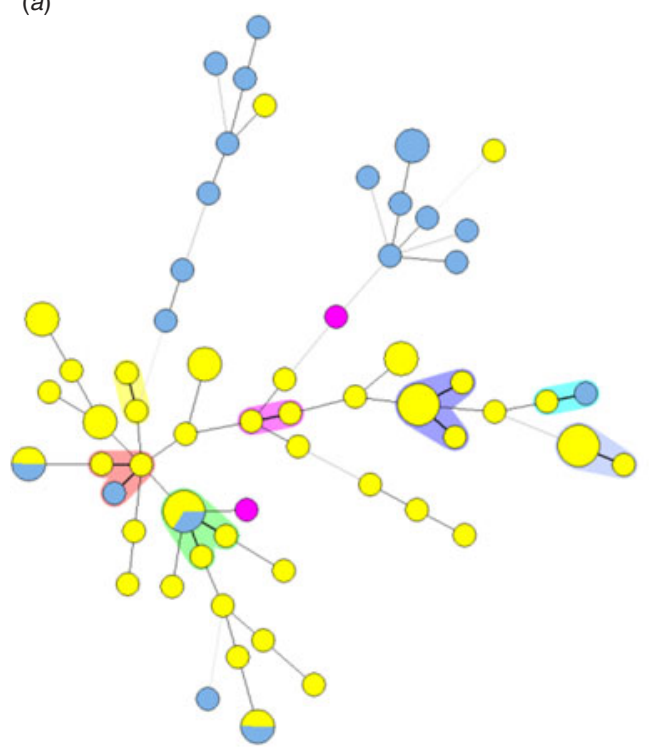

(b)

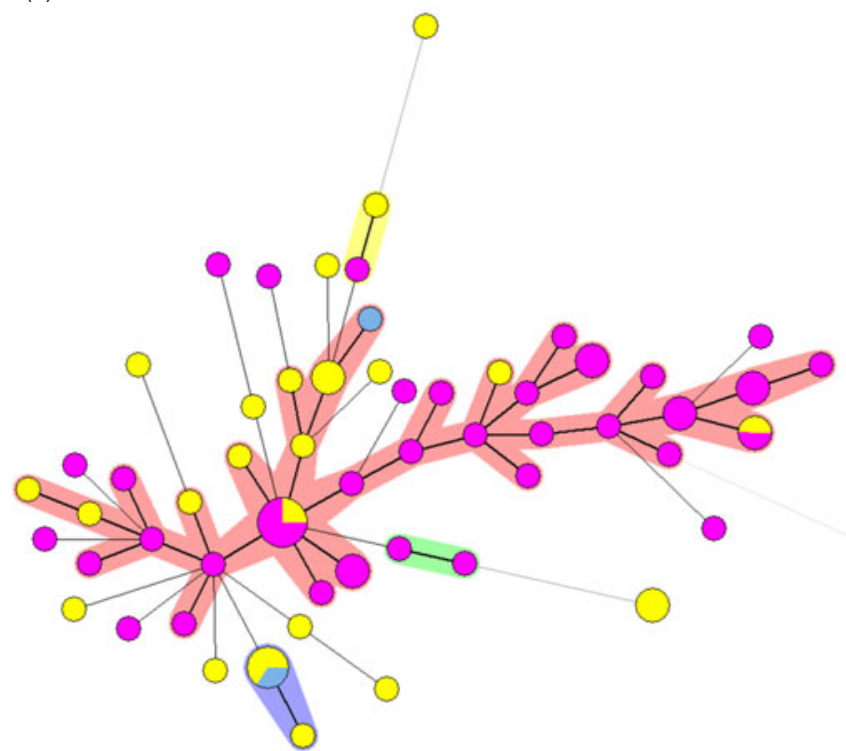

Fig. 4. Multi-locus sequence typing (MLST) of Campylobacter isolated from animals in Dong Thap province, Mekong delta, Vietnam. (a) Minimum spanning tree of 73 C. jejuni isolates from animals in Dong Thap province, calculated by sequencing of the 7-target MLST genes. Each individual sequence type is distinguished by separate circles and linked by lines indicating allelic variation. The colour of each sequence type signifies from which animal species each bacterial strain was isolated: blue, ducks; yellow, chickens; pink, pigs. Background shading highlights clonal complexes. (b) Minimum spanning tree of $70 \mathrm{C}$. coli isolated from animals in Dong Thap province, calculated by sequencing of the 7-target MLST genes. Each individual sequence type is distinguished by separate circles and linked by lines indicating allelic variation.

using hybrid and local breeds, and are normally slaughtered at around 10-12 weeks (compared to 5-6 weeks in typical broiler production) [35]. In addition, consumption of older laying hens is also common in backyard production. The lower stocking densities of low-intensity production systems may also explain these differences. In contrast, the observed Campylobacter prevalence in pigs $(53.7 \%)$ was only marginally lower than the overall prevalence compiled from 20 studies on Campylobacter in pigs worldwide $(69 \cdot 7 \%)$. We can also report an important age effect associated with the prevalence of Campylobacter in pigs. Our data also demonstrated low-level of clustering of Campylobacter on farms, predicting that most production units would test positive for Campylobacter if sufficient numbers of animals were tested. This is probably a reflection of limited biosecurity, a mix of species and variable animal ages on farms.

Ninety-nine per cent of the culture isolates were C. jejuni or C. coli, the two main Campylobacter species responsible for clinical cases of diarrhoea in Vietnam [15]. C. jejuni was the most common species in pigs and poultry. An unexpectedly high prevalence of $C$. jejuni was observed in pigs in the district (Chau Thanh) with the highest pig production. Studies have shown that colonization of pigs with $C$. jejuni is common in animals reared outdoors [36]. This suggests that pigs may be an important source of $C$. jejuni in Vietnam, and merits further investigation.

The observed Campylobacter species distribution in chickens differs from a 2005-2006 study in Ho Chi Minh City (HCMC), where $73.9 \%$ of chicken meat samples were identified as C. lari [37]. C. lari was also identified in chicken carcasses from markets sampled in neighbouring Cambodia $(21 \%$ of all isolates) [38]. This discrepancy may reflect differences in the sources of chickens, since it is likely that a larger fraction of birds sold in HCMC are representative of chickens produced under industrial conditions. Despite the high potential for inter-species transmission on the farms, we found no statistical difference in the prevalence of $C$. jejuni between pigs kept on farms that raised and did not raise poultry; similarly, there was no difference in risk of $C$. coli infection in poultry that were and were not exposed to pigs. In contrast, we found an association between presence of $C$. coli in ducks and high density of pigs at the 
commune level, and genotypic evidence of strain transfer between pigs and chickens. Duck farming in the Mekong delta is characterized by low levels of biosecurity and itinerant grazing practices [39], which are likely to expose ducks at risk of infection when they graze in areas where pig effluent is used. Furthermore, the presence of multiple flocks of ducks on a single farm was associated with an increased prevalence of Campylobacter, similar to an observation from broiler operations [40].

Our results indicate a high level of antimicrobial resistance in Campylobacter isolates. This is a particular a concern for erythromycin (100\% resistance) and ciprofloxacin ( $21 \%$ resistance), two antimicrobials commonly used to treat serious Campylobacter infections. We found high rates of antimicrobial usage on farms, with a wide range of antimicrobial compounds, suggesting a sustained selective pressure inducing the potential for the development of antimicrobial resistance. In a previous study by Garin et al. a higher prevalence of resistance to ciprofloxacin $(95 \%)$ but a lower prevalence to erythromycin (25\%) was reported in isolates from chickens [37]. Antimicrobial resistance in human Campylobacter isolates from Vietnam from children with diarrhoea presenting to healthcare settings in HCMC has been increasing over recent years and current isolates show almost identical antimicrobial resistance patterns to those presented in this study (data not shown). Data from 88 human isolates from 1996 to 1999 indicate that 7\% of isolates were resistance to nalidixic acid and ciprofloxacin and all strains were sensitive to azithromycin [41]. A likely explanation for this is the increased use of antimicrobials in human and veterinary medicine over the same period $[42,43]$.

The last few years have seen the establishment of a large number of integrated broiler, commercial chicken layer, and pig companies in Vietnam which supply meat to the increasing population. As a consequence of increasing demand, these production systems are likely to become common in future years. Except for the large pig units in Chau Thanh district, industrial size operations are still uncommon in Dong Thap province, but this is changing. It is likely that industrialized production systems will contribute to a higher burden of Campylobacter organisms in the food chain, due to increased stocking density and stress, homogenization of diet, and reduced levels of immunity [44]. In addition, changes associated with urbanization, improved sanitation and water quality are likely to reduce the exposure to bacterial pathogens during childhood, reducing levels of population immunity. Something similar has been observed in China, where an increase in Campylobacter cases in older age groups has been observed [45]. This combination of increased exposure through food and reduced population immunity is likely to contribute in an increased burden of human Campylobacter infections throughout the next decade.

We conclude that Campylobacter, an important cause of diarrhoea in children, is highly prevalent in animal production systems in Vietnam. We predict that intensification of animal production systems and increased urbanization will result in a further increase in the incidence of this infection and a change in the epidemiology in animals and humans. Given the high levels of resistance to antimicrobials in these organisms it is imperative to improve the surveillance and control of Campylobacter throughout the length of the food chain. Future interventions targeting a reduction in the prevalence of Campylobacter in farmed animal populations in Vietnam will become increasingly important.

\section{SUPPLEMENTARY MATERIAL}

For supplementary material accompanying this paper visit http://dx.doi.org/10.1017/S0950268813002410.

\section{ACKNOWLEDGEMENTS}

We thank the Sub-Department of Animal Health of Dong Thap for their support. We are indebted to Dr Marcel Wolbers (OUCRU) for statistical advice. This work has been co-funded by ZoNMW/ WOTRO (The Netherlands), VIBRE Project (No. 205100012) and the Vietnam Initiative on Zoonotic Infections (VIZIONS), part of the Wellcome Trust Major Overseas Programme (UK).

\section{DECLARATION OF INTEREST}

None.

\section{REFERENCES}

1. Silva J, et al. Campylobacter spp. as a foodborne pathogen: a review. Frontiers in Food Microbiology 2011; 2: 200.

2. WHO. Campylobacter: Fact sheet no. 255, 2011. (http:// www.who.int/mediacentre/factsheets/fs255/en/index.html). Accessed 4 October 2012. 
3. Coker AO, et al. Human campylobacteriosis in developing countries. Emerging Infectious Diseases 2002; 8: $237-244$.

4. Hermans D, et al. Poultry as a Host for the Zoonotic Pathogen Campylobacter jejuni. Vector-Borne and Zoonotic Diseases 2012; 12: 89-98.

5. Fosse J, Seegers H, Magras C. Prevalence and risk factors for bacterial food-bornezoonotic hazards in slaughter pigs: a review. Zoonoses and Public Health 2009; 56: 429-454.

6. Chatre $\mathbf{P}$, et al. Prevalence and antimicrobial resistance of Campylobacter jejuni and Campylobacter coli isolated from cattle between 2002 and 2006 in France. Journal of Food Protection 2010; 73: 825-831.

7. Humphrey T, O'Brien S, Madsen M. Campylobacters as zoonotic pathogens: a food production perspective. International Journal of Food Microbiology 2007; 117: 237-257.

8. Olson C, et al. Epidemiology of Campylobacter jejuni infections in industrialised nations. In: Irving $\mathrm{N}$, Szymanski C, Blaser M, eds. Campylobacter. Washington, DC: ASM Press, 2008, pp. 163-189.

9. Domingues AR, et al. Source attribution of human campylobacteriosis using a meta-analysis of casecontrol studies of sporadic infections. Epidemiology and Infection 2012; 140: 970-981.

10. Sheppard SK, et al. Campylobacter genotyping to determine the source of human infection. Clinical Infectious Diseases 2009; 48: 1072-1078.

11. Horrocks SM, et al. Incidence and ecology of Campylobacter jejuni and coli in animals. Anaerobe 2009; 15: 18-25.

12. Anon. Analysis of the baseline survey on the prevalence of Campylobacter in broiler batches and of Campylobacter and Salmonella on broiler carcasses in the EU, 2008: Part A: Campylobacter and Salmonella prevalence estimates. EFSA Journal 2010; 8: 1503.

13. Lee MD, Newell DG. Campylobacter in poultry: Filling an ecological niche. Avian Diseases 2006; 50: 1-9.

14. Adzitey F, Huda N, Ali GR. Prevalence and antibiotic resistance of Campylobacter, Salmonella, and L. monocytogenes in ducks: a review. Foodborne Pathogens and Disease 2012; 9: 498-505.

15. Isenbarger D, et al. Prospective study of the incidence of diarrhoea and prevalence of bacterial pathogens in a cohort of Vietnamese children along the Red River. Epidemiology and Infection 2001; 127: 229-236.

16. Hien BT, et al. Diarrhoeagenic Escherichia coli and other causes of childhooddiarrhoea: a case-control study in children living in a wastewater-use area in Hanoi, Vietnam. Journal of Medical Microbiology 2007; 56: 1086-1096.

17. Bodhidatta $\mathbf{L}$, et al. Rotavirus disease in young children from Hanoi, Vietnam. Pediatric Infectious Disease Journal 2007; 26: 325-328.

18. Ha TA, Pham TY. Study of Salmonella, Campylobacter, and Escherichia coli contamination in raw food available in factories, schools, and hospital canteens in Hanoi, Vietnam. Annals of the New York Academy of Sciences 2006; 1081: 262-265.
19. Luu QH, et al. Study on the prevalence of Campylobacter spp. from 361 chicken meat in 362 Hanoi, Vietnam. Annals of the New York Academy of Sciences 2006; 1081: 273-275.

20. Hwang MN, Ederer GM. Rapid hippurate hydrolysis method for presumptive identification of group B streptococci. Journal of Clinical Microbiology 1975; 1: 114-115.

21. Linton D, et al. PCR detection, identification to species level, and fingerprinting of Campylobacter jejuni and Campylobacter coli direct from diarrheic samples. Journal of Clinical Microbiology 1997; 35: 2568-2572.

22. Persson S, Olsen KE. Multiplex PCR for identification of Campylobacter coli and Campylobacter jejuni from pure cultures and directly on stool samples. Journal of Medical Microbiology 2005; 54: 1043-1047.

23. Dingle KE, et al. Multilocus sequence typing system for Campylobacter jejuni. Journal of Clinical Microbiology 2001; 39: 14-23.

24. Dohoo I, Martyn W, Stryhn H. Veterinary Epidemiologic Research, 1st edn. Charlottetown: AVC Inc., 2003.

25. Golstein H, Browne H, Rasbash J. Partitioning variation in multilevel models. Understanding Statistics 2002; 1: 223-231.

26. Hosmer DW, Lemeshow S. Applied Logistic Regression, 1st edn. New York: Wiley-Interscience, 1989.

27. Burnham KP, Anderson DR. Model Selection and Multimodel Inference: A Practical Information-Theoretic Approach, 2nd edn, London: Springer-Verlag, 2002.

28. Saleha A. Isolation and characterization of Campylobacter jejuni from broiler chickens in Malaysia. International Journal of Poultry Science 2002; 1: 94 97.

29. Mansouri-Najand L, Saleha AA, Wai SS. Prevalence of multidrug resistance Campylobacter jejuni and Campylobacter coli in chickens slaughtered in selected markets, Malaysia. Tropical Biomedicine 2012; 29: 231-238.

30. Padungtod P, Kaneene JB. Campylobacter in food animals and humans in Northern Thailand. Journal of Food Protection 2005; 68: 2519-2526.

31. Bouwknegt $\mathbf{M}$, et al. Risk factors for the presence Campylobacter spp. in Dutch broiler flocks. Preventive Veterinary Medicine 2004; 62: 35-49.

32. Gregory E, et al. Epidemiological study of Campylobacter spp. in broilers: source, time of colonization, and prevalence. Avian Diseases 1997; 41: 890-898.

33. Lindblom GB, Sjörgren E, Kaijser B. Natural Campylobacter colonization in chickens raised under different environmental conditions. Journal of Hygiene (London) 1986; 96: 385-391.

34. Kalupahana R, et al. Colonization of Campylobacter in broiler chickens and layer hens reared in tropical climates with low-biosecurity. Applied of Environmental Microbiology 2013; 79: 393-395.

35. FAO. Poultry production systems in Viet Nam. GCP/ RAS/228/GER Working Paper No. 4. Rome. 2008.

36. Jensen AN, et al. The occurrence and characterization of Campylobacter jejuni and C. coli in organic pigs and 
their outdoor environment. Veterinary Microbiology 2006; 1-3: 96-105.

37. Garin B, et al. Prevalence, quantification and antimicrobial resistance of Campylobacter spp. on chicken neckskins at points of slaughter in 5 major cities located on 4 continents. International Journal of Food Microbiology 2012; 157: 102-107.

38. Lay KS, et al. Prevalence, numbers and antimicrobial susceptibilities of Salmonella serovars and Campylobacter spp. in retail poultry in Phnom Penh, Cambodia. Journal of Veterinary Medical Science 2011; 73: 325-329.

39. Minh PQ, et al. A description of the management of itinerant grazing ducks in the Mekong river delta of Vietnam. Preventive Veterinary Medicine 2010; 94: 101-107.

40. Henry I, et al. Prevalence and risk factors for Campylobacter spp. in chicken broiler flocks in Reunion Island (Indian Ocean). Preventive Veterinary Medicine 2011; 100: 64-70.

41. Isenbarger DW, et al. Comparative antibiotic resistance of diarrheal pathogens from 410 Vietnam and Thailand,
1996-1999. Emerging Infectious Diseases 2002; 8: $175-180$.

42. Hoa NQ, et al. Unnecessary antibiotic use for mild acute respiratory infections during 28-day follow-up of 823 children under five in rural Vietnam. Transactions of the Royal Society of Tropical Medicine and Hygiene 2011; 105: 628-636.

43. GARP. Situation analysis: antibiotic use and resistance in Vietnam. Washington, DC: Center for Disease Dynamics, Economics and Policy, 2010 (http://www. cddep.org/sites/cddep.org/files/publication_files/

VN_Report_web_1.pdf). Accessed 17 October 2012.

44. Graham JP, et al. The animal-human interface and infectious disease in industrial food animal production: rethinking biosecurity and biocontainment. Public Health Reports 2008; 123: 282-299.

45. Hou FQ, Sun XT, Wang GQ. Clinical manifestations of Campylobacter jejuni infection in adolescents and adults, and change in antibiotic resistance of the pathogen over the past 16 years. Scandinavian Journal of Infectious Diseases 2012; 44: 439-443. 